\title{
自适应权重多视角度量学习的遥感图像场景分类方法
}

\author{
周国华 ${ }^{1,2,3)}$ ，蒋晖 ${ }^{1,2)}$ ，顾晓清 ${ }^{2}$ ，殷新春 ${ }^{3)}$ \\ 1) (常州工业职业技术学院信息工程学院 常州 213164) \\ 2) (常州大学计算机与人工智能学院 常州 213164) \\ 3) (扬州大学信息工程学院 扬州 225127) \\ (tiddyddd@sina.com.cn)
}

\begin{abstract}
摘 要: 遥感图像易受光照和气象条件等干扰因素的影响, 且随着遥感设备分辨率的提高, 遥感图像中出现了更多 的地表细节的问题. 为了提高遥感图像的场景分类的准确度, 提出一种自适应权重多视角度量学习方法. 首先使用 多个视角下的数据特征学习具有分辨力的度量空间, 使在度量空间内同类图像紧凑, 异类图像尽可能地远离; 然后 引人权重向量, 在度量学习的过程中自适应地调节各视角间的权重关系; 最后利用核技巧扩展至非线性空间, 更有 效地挖掘隐藏于视角间的关联和互补信息. 在 Google 和 WHU-RS 遥感图像数据集上的实验结果表明, 该方法具有良 好的分类性能, 平均分类准确率分别达到 90.26\%和 92.62\%, 显著优于对比的单视角和多视角分类方法.
\end{abstract}

关键词：遥感图像场景分类; 自适应权重; 多视角学习; 度量学习

中图法分类号: TP391.41

DOI: $10.3724 /$ SP.J.1089.2021.18571

\section{Self-Weighted Multi-View Metric Learning and Its Application for Remote Sensing Image Scene Classification}

\author{
Zhou Guohua $^{1,2,3)}$, Jiang Hui ${ }^{1,2)}$, Gu Xiaoqing ${ }^{2)}$, and Yin Xinchun ${ }^{3)}$ \\ 1) (College of Information Engineering, Changzhou Institute of Industry Technology, Changzhou 213164) \\ ${ }^{2)}$ (School of Computer Science and Artificial Intelligence, Changzhou University, Changzhou 213164) \\ 3) (College of Information Engineering, Yangzhou University, Yangzhou 225127)
}

\begin{abstract}
The remote sensing images are susceptible to interference factors such as illumination and meteorological conditions, and with the improvement of the resolution of remote sensing equipment, more surface details appear in the remote sensing image. In order to improve the accuracy of remote sensing image scene classification, a self-weighted multi-view metric learning (SW-MVML) method is proposed. Firstly, data features from multiple views are used to learn a discriminative metric space, which makes the similar images compact and dissimilar images as far away as possible. Then, the weight vector is introduced to adaptively adjust the weight relationship among different views. Finally, the kernel technique is used to extend the method to non-linear space, so that the correlation and complementary information hidden between views can be effectively exploited. The experimental results on Google and WHU-RS remote sensing image datasets show that the proposed method achieves good classification performance with the average classification accuracy of $90.26 \%$ and $92.62 \%$ respectively, which is significantly better than the comparative single-view and multi-view classification methods.
\end{abstract}

收稿日期：2020-08-12；修回日期：2021-03-11. 基金项目：国家自然科学基金(61472343，61806026); 江苏省自然科学基金 (BK20180956); 江苏省高职院校教师专业带头人高端研修项目(2020GRGDYX059). 周国华(1977一), 男, 硕士, 副教授, CCF 高级会 员, 主要研究方向为智能学习、模式识别; 蒋晖(1971一), 男, 硕士, 副教授, 主要研究方向为信息化智能处理; 顾晓清(1981一), 女, 博士, 副教授, 硕士生导师, 主要研究方向为模式识别; 殷新春(1962-), 男, 博士, 教授, 博士生导师, CCF 高级会员, 主要研究方 向为人工智能、密码学. 
Key words: remote sensing image classification; self-weight; multi-view learning; metric learning

近年来，随着遥感技术的发展，遥感卫星成像 技术已广泛应用于农业、地质、林业和区域规划等 领域，用于分析和管理自然资源和人类活动 ${ }^{[1-3]}$. 其中，遥感图像的场景分类受到广泛关注，因为场 景分类与人类存在有关的活动密切相关. 不同于 一般计算机视觉任务中的自然图像(如人脸识别), 遥感图像的场景分类通常具有复杂的地面物体排 列，其包含的内在信息更丰富. 例如，物体可能以 不同的尺度和方向出现、对象数量(密集的居住场 景和稀疏的居住场景)以及这些对象之间的空间关 系等，这些信息容易导致场景分类出现类内差异 大且类间差异小的问题 ${ }^{[4]}$. 因此，场景分类的关键 问题是如何提取有效的特征并提高分类的准确率.

目前常用于遥感图像特征提取方法有颜色、纹 理、空间信息和光谱信息等. 例如，尺度不变特征 变换(scale-invariant feature transform, SIFT)和局部 二值模式(local binary patterns, LBP)是纹理特征提 取的代表 ${ }^{[5-6]}$. 许多算法在空间共生和空间关系金 字塔中, 通常使用 SIFT 特征进行遥感图像的场景 分类 ${ }^{[7]}$. LBP 常与主成分分析和多尺度信息等方法 相结合来表示场景图像 ${ }^{[8]}$. 但遥感图像的信息量往 往与其种类分布密切相关. 例如, 沙漠和草地类图 像的信息量与商业区和港口类图像差别很大，因 此传统的各类浅层次的信息不能满足遥感场景分 类的需要. 另外, 这些特征提取方法也不能适应多 种特征的复杂组合, 当某些类别对应的特征不够 显著时, 会降低后续分类器性能.

基于一致性和互补性原理，多视角学习是利 用事物的多种视角表征进行建模求解的一种机器 学习方法 ${ }^{[9]}$. 多视角学习方法可以综合利用从多途 径或多层次获取的一组数据特征, 这些独立而多 样的特征可以分别从遥感场景的纹理、结构和颜色 信息, 以及多层次特征中提取出来. 因为多视角数 据的每个视角可能包含其他视角中不可用的特定 信息, 多视角学习可以从多个视角出发, 同时利用 多种特征提取方法获得的遥感图像信息，从而提 高遥感图像场景分类的性能 ${ }^{[10]}$. 多视角学习常采 用多核学习和子空间学习 2 种手段. 多核学习将不 同视角数据采用线性或非线性的核函数映射到不 同的特征空间，并对映射到不同空间的特征进行 融合; 子空间学习通过学习一个潜在于多个视角 的共享隐空间，在该共享隐空间中可以更好地利 用不同视角的数据. 例如, Luus 等 ${ }^{[11]}$ 提出一种基于
多视角深度学习的方法, 使用多尺度输人策略进 行多光谱土地分类. Yang 等 ${ }^{[12]}$ 提出一种双视角相 关分析网络, 并将其扩展到多视角学习模型, 利用 叠层多视角滤波器组来构造深卷积结构. 为减少空 间/辐射分辨率的差异和不同视角下的变化, Lefèvre 等 ${ }^{[13]}$ 提出一种无缝多视角的场景分析方法, 将从地 面和俯视获取的图像相结合, 并使用多视角图像完 成从卫星到街道的场景重建和分类. 刘涁等 ${ }^{[14]}$ 提出 基于 2 个视角的 LM(Levenberg-Marquard)优化的同 步配准影像拼接算法, 使用同步配准方式实现由 2 个视角图片配准合并到全区. 吴芳青等 ${ }^{[15]}$ 提出一 种利用遥感图像几何结构的多视角图像配准算法, 利用全局与局部几何结构特征的互补提高多视角 遥感图像配准的准确率.

为了有效地利用遥感场景图像的特征并提高 遥感场景分类的准确度, 本文提出一种自适应权 重多视角度量学习(self-weighted multi-view metric learning, SW-MVML)方法. 该方法基于分类目的 在多个视角之间学习具有分辨能力的度量空间, 利用不同视角特征的多样性和互补性, 使同类图 像在度量空间紧凑, 异类图像在度量空间尽可能 地分离. 然后, 引人权重向量对各视角进行自适应 加权处理, 在度量矩阵学习过程中有效地调节各 视角间的权重关系. 最后, 利用核技巧将本文方法 扩展至非线性空间，有效地挖掘隐藏于视角间的关 联和互补信息, 使方法具有更强的判别能力. 最后, 在 2 个通用遥感图像数据集上验证本文方法的效果. 与传统的多视角度量方法相比, 文献[13,16-17]使 用欧几里得度量衡量不同样本间的距离, 无法考 虑不同特征间的关联; 本文方法使用马氏距离计 算样本间的协方差矩阵进行度量, 能充分地考虑 样本不同维度的特征之间的关联. 另外, 虽然近年 来深度学习方法在许多计算机视觉任务中获得有 效的描述, 但在标记样本数量有限的情况下, 深度 学习对高维遥感图像的分类效果往往不能令人满 意, 而且深度学习模型需要额外的硬件支持, 时间 复杂度高, 训练时间较长.

\section{1 相关工作}

在计算机视觉领域, 度量学习指利用给定的 图像对来计算特征向量对之间的距离. 度量学习 一般会考虑使用距离度量, 以常用的马氏距离 ${ }^{[18]}$ 
为例，给定数据集 $\left\{\boldsymbol{x}_{i}\right\}_{i=1}^{N} \subset \mathbb{R}^{d} ， \boldsymbol{x}_{i}$ 表示输人空间 的第 $i$ 个样本, 2 幅图像 $\boldsymbol{x}_{i}$ 和 $\boldsymbol{x}_{j}$ 之间的距离度量可 以写为

$$
d_{M}^{2}\left(x_{i}, x_{j}\right)=\left\|x_{i}-x_{j}\right\|_{M}^{2}=\left(x_{i}-x_{j}\right)^{\mathrm{T}} M\left(x_{i}-x_{j}\right)
$$

其中, $\boldsymbol{M}$ 是半正定矩阵, 可以分解为 $\boldsymbol{M}=\boldsymbol{H} \boldsymbol{H}^{\mathrm{T}}$, 矩阵 $\boldsymbol{H}^{d \times m}(m \leqslant d)$ 为度量矩阵. 则式(1)可表示为

$$
d_{\boldsymbol{M}}^{2}\left(\boldsymbol{x}_{i}, \boldsymbol{x}_{j}\right)=\left(\boldsymbol{H} \boldsymbol{x}_{i}-\boldsymbol{H} \boldsymbol{x}_{j}\right)^{\mathrm{T}}\left(\boldsymbol{H} \boldsymbol{x}_{i}-\boldsymbol{H} \boldsymbol{x}_{j}\right)
$$

因此，度量学习的本质是学习一个映射空间， 同类图像对输出接近零的正值, 异类图像对输出 较大的值.

\section{2 自适应权重多视角度量学习方法}

\section{1 目标函数构建}

为了提高遥感场景分类的准确率，本文利用 遥感场景图像的不同特征构造的数据进行分析, 每个特征的数据样本视为一个视角, 将多个视角 的协同工作与度量学习结合在一起求得一个具有 分辨能力的特征空间，在最小化同类图像对间的 距离的同时, 最大化异类图像对间的距离. 各视角 在协同学习时应能够充分挖掘视角间的相似信息, 以获得全面且稳定的分类结果. 为此, 本文引人权 重向量对各视角的效用进行自适应加权处理，在 学习过程中有效地调节各视角间的权重关系，使 最具有分辨能力的视角的权重尽可能大，分辨能 力模糊的视角的权重尽可能小. 特别地, 将上述技 术扩展至非线性空间，进一步增强本文方法的判 别能力.

假设根据不同特征提取方法得到的 $L$ 个多视 角图像数据集为 $\left\{\boldsymbol{X}^{1}, \boldsymbol{X}^{2}, \cdots, \boldsymbol{X}^{L}\right\}$, 其中, $\boldsymbol{X}^{l}$ 为第 $l$ 个视角下的数据子集; $\boldsymbol{x}_{i}^{l}$ 表示样本 $\boldsymbol{x}_{i}$ 在第 $l$ 个视 角下的特征向量. 首先根据样本的空间分布定义 2 个图像对数据集分别为

$$
\begin{aligned}
& \boldsymbol{S}=\left\{\left(\boldsymbol{x}_{i}, \boldsymbol{x}_{j}\right) \mid \boldsymbol{x}_{i} \text { 和 } \boldsymbol{x}_{j} \text { 同类 }\right\} \\
& \boldsymbol{D}=\left\{\left(\boldsymbol{x}_{i}, \boldsymbol{x}_{j}\right) \mid \boldsymbol{x}_{i} \text { 和 } \boldsymbol{x}_{j} \text { 异类 }\right\}
\end{aligned}
$$

通过度量学习一个具有分辨能力的映射空间, 使同类图像尽可能接近, 异类图像尽可能远离, 即 在度量空间中有更紧凑的类内距离和更可分的类 间距离. 使用每个视角的类内散度和类间散度分 别描述类内的紧凑性和类间的可分性. 第 $l$ 个视角 的类间和类内散度分别为

$$
\boldsymbol{S}^{l}=\frac{1}{2|\boldsymbol{S}|} \sum_{i, j=1}^{|\boldsymbol{S}|} \operatorname{tr}\left(\boldsymbol{H}^{\mathrm{T}}\left(\boldsymbol{x}_{i}^{l}-\boldsymbol{y}_{j}^{l}\right)\left(\boldsymbol{x}_{i}^{l}-\boldsymbol{y}_{j}^{l}\right)^{\mathrm{T}} \boldsymbol{H}\right)
$$

$$
\boldsymbol{D}^{l}=\frac{1}{2|\boldsymbol{D}|} \sum_{i, j=1}^{|\boldsymbol{D}|} \operatorname{tr}\left(\boldsymbol{H}^{\mathrm{T}}\left(\boldsymbol{x}_{i}^{l}-\boldsymbol{y}_{j}^{l}\right)\left(\boldsymbol{x}_{i}^{l}-\boldsymbol{y}_{j}^{l}\right)^{\mathrm{T}} \boldsymbol{H}\right)
$$

其中, $\boldsymbol{S}^{l}$ 和 $\boldsymbol{D}^{l}$ 分别表示在第 $l$ 个视角下的同类图像 对集合和异类图像对集合; $|\boldsymbol{S}|$ 和 $|\boldsymbol{D}|$ 分别表示 $\boldsymbol{S}^{l}$ 和 $D^{l}$ 中图像对的数量.

本文采用多视角学习框架，利用多个视角间 的关联性和差异性, 通过学习不同数据特征的互 补信息使所提方法更具区分性. 因此，本文方法的 目标式可以表示为

$$
\left\{\begin{array}{l}
\min _{\boldsymbol{H}, \boldsymbol{\Delta}} \sum_{l=1}^{L} \Delta_{l}\left(\frac{1}{2|\boldsymbol{S}|} \sum_{i, j=1}^{|\boldsymbol{S}|} \operatorname{tr}\left(\boldsymbol{H}^{\mathrm{T}}\left(\boldsymbol{x}_{i}^{l}-\boldsymbol{y}_{j}^{l}\right)\left(\boldsymbol{x}_{i}^{l}-\boldsymbol{y}_{j}^{l}\right)^{\mathrm{T}} \boldsymbol{H}\right)-\right. \\
\left.\frac{1}{2|\boldsymbol{D}|} \sum_{i, j=1}^{|\boldsymbol{D}|} \operatorname{tr}\left(\boldsymbol{H}^{\mathrm{T}}\left(\boldsymbol{x}_{i}^{l}-\boldsymbol{y}_{j}^{l}\right)\left(\boldsymbol{x}_{i}^{l}-\boldsymbol{y}_{j}^{l}\right)^{\mathrm{T}} \boldsymbol{H}\right)\right) \\
\text { s.t. } \boldsymbol{H}^{\mathrm{T}} \boldsymbol{H}=\boldsymbol{I}, \sum_{l=1}^{L} \Delta_{l}=1, \Delta_{l} \geqslant 0
\end{array}\right.
$$

其中, $\boldsymbol{\Delta}=\left[\Delta_{l}, \Delta_{2}, \cdots, \Delta_{L}\right]$ 为权重向量, 其分量 $\Delta_{l}$ 表 示各视角的重要程度; $I$ 为单位矩阵. 值得注意的 是, 向量 $\boldsymbol{\Delta}$ 的最优值不是通过人为设定, 而是作为 一个未知量求解得到. 显然, 视角 $l$ 在学习投影矩 阵 $\boldsymbol{H}$ 时的作用越大, 视角 $l$ 越重要. 当 $\Delta_{l} \rightarrow 0$ 时, 表示视角 $l$ 的特征对于判别任务的学习是无用的, 此时式(7)是一个单视角学习问题. 然而, 也可以 看到, 在极端条件下会出现某个视角占绝对主导, 即 $\Delta l \rightarrow 1$ 的情况, 此时, 其他视角几乎不起作用. 为了避免这一情况, 引入模糊指数 $r(r>1)$, 将权重 $\Delta$, 改写为 $\Delta_{l}^{r}$, 目标式可以进一步表示为

$$
\left\{\begin{array}{l}
\min _{\boldsymbol{H}, \boldsymbol{\Delta}} \sum_{l=1}^{L} \Delta_{l}^{r}\left(\frac{1}{2|\boldsymbol{S}|} \sum_{i, j=1}^{|\boldsymbol{S}|} \operatorname{tr}\left(\boldsymbol{H}^{\mathrm{T}}\left(\boldsymbol{x}_{i}^{l}-\boldsymbol{y}_{j}^{l}\right)\left(\boldsymbol{x}_{i}^{l}-\boldsymbol{y}_{j}^{l}\right)^{\mathrm{T}} \boldsymbol{H}\right)-\right. \\
\left.\frac{1}{2|\boldsymbol{D}|} \sum_{i, j=1}^{|\boldsymbol{D}|} \operatorname{tr}\left(\boldsymbol{H}^{\mathrm{T}}\left(\boldsymbol{x}_{i}^{l}-\boldsymbol{y}_{j}^{l}\right)\left(\boldsymbol{x}_{i}^{l}-\boldsymbol{y}_{j}^{l}\right)^{\mathrm{T}} \boldsymbol{H}\right)\right) \\
\text { s.t. } \boldsymbol{H}^{\mathrm{T}} \boldsymbol{H}=\boldsymbol{I}, \sum_{l=1}^{L} \Delta_{l}=1, \Delta_{l} \geqslant 0
\end{array}\right.
$$

设

$$
\begin{aligned}
& \boldsymbol{A}^{l}=\frac{1}{2|\boldsymbol{S}|} \sum_{i, j=1}^{|\boldsymbol{S}|}\left(\boldsymbol{x}_{i}^{l}-\boldsymbol{y}_{j}^{l}\right)^{\mathrm{T}}\left(\boldsymbol{x}_{i}^{l}-\boldsymbol{y}_{j}^{l}\right), \\
& \boldsymbol{B}^{l}=\frac{1}{2|\boldsymbol{D}|} \sum_{i, j=1}^{|\boldsymbol{D}|}\left(\boldsymbol{x}_{i}^{l}-\boldsymbol{y}_{j}^{l}\right)^{\mathrm{T}}\left(\boldsymbol{x}_{i}^{l}-\boldsymbol{y}_{j}^{l}\right),
\end{aligned}
$$

式(8)可以简写为

$$
\left\{\begin{array}{l}
J(\boldsymbol{H}, \boldsymbol{\Delta})=\min _{\boldsymbol{H}, \boldsymbol{\Delta}} \operatorname{tr}\left(\boldsymbol{H}^{\mathrm{T}}\left(\sum_{l=1}^{L} \Delta_{l}^{r}\left(\boldsymbol{A}^{l}-\boldsymbol{B}^{l}\right)\right) \boldsymbol{H}\right) \\
\text { s.t. } \boldsymbol{H}^{\mathrm{T}} \boldsymbol{H}=\boldsymbol{I}, \sum_{l=1}^{L} \Delta_{l}^{r}=1, \Delta_{l} \geqslant 0
\end{array}\right.
$$




\section{2 方法求解}

式(9)的优化问题是一个非线性约束的非凸问 题. 本文方法涉及 2 个待求解参数 $\{\boldsymbol{H}, \boldsymbol{A}\}$, 使用迭 代优化策略交替求解这 2 个参数. 首先固定参数 $\boldsymbol{A}$ 求解 $\boldsymbol{H}$, 式(9)可以表示为

$$
\left\{\begin{array}{l}
\min _{\boldsymbol{H}, \boldsymbol{\Delta}} \operatorname{tr}\left(\boldsymbol{H}^{\mathrm{T}}\left(\sum_{l=1}^{L} \Delta_{l}^{r}\left(\boldsymbol{A}^{l}-\boldsymbol{B}^{l}\right)\right) \boldsymbol{H}\right) \\
\text { s.t. } \boldsymbol{H}^{\mathrm{T}} \boldsymbol{H}=\boldsymbol{I}
\end{array}\right.
$$

矩阵 $\boldsymbol{H}$ 的求解可以转化为一个特征值分解问题

$$
\left(\sum_{l=1}^{L} \Delta_{l}^{r}\left(\boldsymbol{A}^{l}-\boldsymbol{B}^{l}\right)\right) \boldsymbol{H}=\lambda \boldsymbol{H}
$$

求解得到矩阵 $\boldsymbol{H}$ 后, 固定矩阵 $\boldsymbol{H}$ 求解 $\boldsymbol{\Delta}$. 在 式(9)中引人拉格朗日乘子 $\alpha$, 式(9)可以写成

$J(\boldsymbol{\Delta}, \alpha)=\sum_{l=1}^{L} \Delta_{l}^{r} \operatorname{tr}\left(\boldsymbol{H}^{\mathrm{T}}\left(\boldsymbol{A}^{l}-\boldsymbol{B}^{l}\right) \boldsymbol{H}\right)-\alpha\left(\sum_{l=1}^{L} \Delta_{l}^{r}-1\right)$

其中, $\Delta_{k}$ 有极值的必要条件为 $\frac{\partial J(\boldsymbol{\Delta}, \alpha)}{\partial \Delta_{k}}=0$, 同时 考虑 $\frac{\partial J(\boldsymbol{\Delta}, \alpha)}{\partial \alpha}=0$, 可以得到

$$
\left\{\begin{array}{l}
r \Delta_{l}^{r-1} \operatorname{tr}\left(\boldsymbol{H}^{\mathrm{T}}\left(\boldsymbol{A}^{l}-\boldsymbol{B}^{l}\right) \boldsymbol{H}\right)-\alpha=0 \\
\sum_{l=1}^{L} \Delta_{l}-1=0
\end{array}\right.
$$

由式(13)可得

$$
\begin{aligned}
& \sum_{l=1}^{L} \Delta_{l}=\sum_{l=1}^{L}\left((\alpha / r) \operatorname{tr}\left(\boldsymbol{H}^{\mathrm{T}}\left(\boldsymbol{A}^{l}-\boldsymbol{B}^{l}\right) \boldsymbol{H}\right)\right)^{1 /(r-1)}= \\
& (\alpha / r)^{1 /(r-1)} \sum_{l=1}^{L}\left(1 / \operatorname{tr}\left(\boldsymbol{H}^{\mathrm{T}}\left(\boldsymbol{A}^{l}-\boldsymbol{B}^{l}\right) \boldsymbol{H}\right)\right)^{1 /(r-1)}=1
\end{aligned}
$$

经过化简可得

$$
(\alpha / r)^{1 /(r-1)}=1 / \sum_{l=1}^{L}\left(1 / \operatorname{tr}\left(\boldsymbol{H}^{\mathrm{T}}\left(\boldsymbol{A}^{l}-\boldsymbol{B}^{l}\right) \boldsymbol{H}\right)\right)^{1 /(r-1)}
$$

将式(15)代人式(13), 可以得到 $\Delta l$ 的解析解为

$$
\Delta_{l}=\frac{\left(1 / \operatorname{tr}\left(\boldsymbol{H}^{\mathrm{T}}\left(\boldsymbol{A}^{l}-\boldsymbol{B}^{l}\right) \boldsymbol{H}\right)\right)^{1 /(r-1)}}{\sum_{l=1}^{L}\left(1 / \operatorname{tr}\left(\boldsymbol{H}^{\mathrm{T}}\left(\boldsymbol{A}^{l}-\boldsymbol{B}^{l}\right) \boldsymbol{H}\right)\right)^{1 /(r-1)}}
$$

基于以上的分析，本文算法步骤如下.

输人. $L$ 个视角的图像对, 模糊指数 $r$, 最大迭 代次数 $t_{\max }$ 和收玫阈值 $\delta$.

输出。度量矩阵 $\boldsymbol{H}$.

初始化. 初始化 $\boldsymbol{\Delta}=[1 / L, 1 / L, \cdots, 1 / L]$, 使用式(11)计 算 $\boldsymbol{H}$
Repeat

$t=t+1$.

固定 $\boldsymbol{\Delta}(t)$ ，使用式(11)计算 $\boldsymbol{H}(t)$.

固定 $\boldsymbol{H}(t)$ ，使用式(16)计算 $\boldsymbol{\Delta}(t)$.

使用式(9)计算目标式 $J(t)$ :

Until $\|J(t)-J(t-1)\| \leqslant \delta$ 或 $t \geqslant t_{\max }$

得到 $\boldsymbol{H}$ 最优解 $\boldsymbol{H}^{*}=\boldsymbol{H}(t)$.

\section{3 非线性方法}

高维遥感图像通常呈现高维非线性的特征 ${ }^{[19]}$, 因此，本节使用核技巧将线性方法拓展至非线性 空间. 利用非线性核函数 $\phi(\cdot)$, 将数据样本投影到 再生核 Hilbert 空间(reproducing kernel Hilbert space, RKHS $)^{[20-21]}$ 中, 样本 $\boldsymbol{x}_{i}$ 和 $\boldsymbol{x}_{j}$ 的内积可以表示为

$$
k\left(\boldsymbol{x}_{i}, \boldsymbol{y}_{j}\right)=\varphi\left(\boldsymbol{x}_{i}\right)^{\mathrm{T}} \varphi\left(\boldsymbol{y}_{j}\right)
$$

在式(17)的基础上，使用核函数 $\phi(\cdot)$ 将线性投 影矩阵 $\tilde{\boldsymbol{H}}=\left[\tilde{\boldsymbol{h}}_{1}, \tilde{\boldsymbol{h}}_{2}, \cdots, \tilde{\boldsymbol{h}}_{r}\right]$ 映射到一个低维特征空间 $\boldsymbol{R}^{r}$, 即 $\tilde{\boldsymbol{H}}^{\mathrm{T}} \varphi(\boldsymbol{x})$. 因此，特征空间 $\mathbb{R}^{r}$ 中马氏距离 可以表示为

$$
\begin{aligned}
& \left\|\varphi\left(\boldsymbol{x}_{i}\right)-\varphi\left(\boldsymbol{y}_{j}\right)\right\|= \\
& \sqrt{\left(\varphi\left(\boldsymbol{x}_{i}\right)-\varphi\left(\boldsymbol{y}_{j}\right)\right)^{\mathrm{T}} \tilde{\boldsymbol{H}} \tilde{\boldsymbol{H}}^{\mathrm{T}}\left(\varphi\left(\boldsymbol{y}_{i}\right)-\varphi\left(\boldsymbol{y}_{j}\right)\right)}
\end{aligned}
$$

设 $\boldsymbol{\varphi}=\left[\varphi\left(\boldsymbol{x}_{1}\right), \varphi\left(\boldsymbol{x}_{2}\right), \cdots, \varphi\left(\boldsymbol{x}_{N}\right)\right], \boldsymbol{\beta}=\left[\beta_{1}, \beta_{2}, \cdots, \beta_{N}\right]^{\mathrm{T}}$ ， 其中, $N$ 为样本的数量. 矩阵 $\tilde{\boldsymbol{h}}$ 和 $\tilde{\boldsymbol{H}}$ 可以表示为

$$
\begin{gathered}
\tilde{\boldsymbol{h}}=\sum_{i=1}^{N} \beta_{i} \varphi\left(\boldsymbol{x}_{i}\right)=\boldsymbol{\varphi} \boldsymbol{\beta} \\
\tilde{\boldsymbol{H}}=\boldsymbol{\varphi} \tilde{\boldsymbol{\beta}} \\
\tilde{\boldsymbol{\beta}}=\left[\boldsymbol{\beta}_{1}, \boldsymbol{\beta}_{2}, \cdots, \boldsymbol{\beta}_{r}\right]
\end{gathered}
$$

根据核技巧，矩阵 $\tilde{\boldsymbol{H}}$ 在空间 $\mathbb{R}^{r}$ 中的投影为

$$
\tilde{\boldsymbol{H}}^{\mathrm{T}} \varphi(\boldsymbol{x})=\tilde{\boldsymbol{\beta}}^{\mathrm{T}} \boldsymbol{\varphi}^{\mathrm{T}} \varphi(\boldsymbol{x})=\tilde{\boldsymbol{\beta}}^{\mathrm{T}} \boldsymbol{K}(\boldsymbol{x})
$$

其中， $\boldsymbol{K}(\boldsymbol{x})=\boldsymbol{\varphi}^{\mathrm{T}} \varphi(\boldsymbol{x})$. 此时，原始度量空间中的 矩阵 $\boldsymbol{S}^{l}$ 和矩阵 $\boldsymbol{D}^{l}$ 在特征空间 $\mathbb{R}^{r}$ 转化为矩阵 $\varphi\left(\boldsymbol{S}^{l}\right)$ 和 $\varphi\left(\boldsymbol{D}^{l}\right) . \varphi\left(\boldsymbol{S}^{l}\right)$ 和 $\varphi\left(\boldsymbol{D}^{l}\right)$ 分别表示为

$$
\begin{aligned}
& \varphi\left(\boldsymbol{S}^{l}\right)=\frac{1}{2|S|} . \\
& \sum_{i, j=1}^{|S|} \tilde{\boldsymbol{\beta}}^{\mathrm{T}}\left(\boldsymbol{K}\left(\boldsymbol{x}_{i}^{l}\right)-\boldsymbol{K}\left(\boldsymbol{y}_{j}^{l}\right)\right)^{\mathrm{T}}\left(\boldsymbol{K}\left(\boldsymbol{x}_{i}^{l}\right)-\boldsymbol{K}\left(\boldsymbol{y}_{j}^{l}\right)\right) \tilde{\boldsymbol{\beta}} \\
& \varphi\left(\boldsymbol{D}^{l}\right)=\frac{1}{2|\boldsymbol{D}|} \cdot \\
& \sum_{i, j=1}^{|\boldsymbol{D}|} \tilde{\boldsymbol{\beta}}^{\mathrm{T}}\left(\boldsymbol{K}\left(\boldsymbol{x}_{i}^{l}\right)-\boldsymbol{K}\left(\boldsymbol{y}_{j}^{l}\right)\right)^{\mathrm{T}}\left(\boldsymbol{K}\left(\boldsymbol{x}_{i}^{l}\right)-\boldsymbol{K}\left(\boldsymbol{y}_{j}^{l}\right)\right) \tilde{\boldsymbol{\beta}}
\end{aligned}
$$

为了简化模型，设 


$$
\begin{aligned}
& \varphi\left(\boldsymbol{A}^{l}\right)=\frac{1}{2|\boldsymbol{S}|} \sum_{i, j=1}^{|\boldsymbol{S}|}\left(\boldsymbol{K}\left(\boldsymbol{x}_{i}^{l}\right)-\boldsymbol{K}\left(\boldsymbol{y}_{j}^{l}\right)\right)^{\mathrm{T}}\left(\boldsymbol{K}\left(\boldsymbol{x}_{i}^{l}\right)-\boldsymbol{K}\left(\boldsymbol{y}_{j}^{l}\right)\right) \\
& \varphi\left(\boldsymbol{B}^{l}\right)=\frac{1}{2|\boldsymbol{D}|} \sum_{i, j=1}^{|\boldsymbol{D}|}\left(\boldsymbol{K}\left(\boldsymbol{x}_{i}^{l}\right)-\boldsymbol{K}\left(\boldsymbol{y}_{j}^{l}\right)\right)^{\mathrm{T}}\left(\boldsymbol{K}\left(\boldsymbol{x}_{i}^{l}\right)-\boldsymbol{K}\left(\boldsymbol{y}_{j}^{l}\right)\right)
\end{aligned}
$$

将 $\varphi\left(\boldsymbol{A}^{l}\right)$ 和 $\varphi\left(\boldsymbol{B}^{l}\right)$ 代人式(10), 由此可得非线性方 法的目标函数为

$$
\left\{\begin{array}{l}
J(\tilde{\boldsymbol{\beta}}, \boldsymbol{\Delta})=\min _{\tilde{\boldsymbol{\beta}}, \Delta} \operatorname{tr}\left(\tilde{\boldsymbol{\beta}}^{\mathrm{T}}\left(\sum_{l=1}^{L} \Delta_{l}^{r}\left(\varphi\left(\boldsymbol{A}^{l}\right)-\varphi\left(\boldsymbol{B}^{l}\right)\right)\right) \tilde{\boldsymbol{\beta}}\right) \\
\text { s.t. } \tilde{\boldsymbol{\beta}}^{\mathrm{T}} \tilde{\boldsymbol{\beta}}=\boldsymbol{I}, \sum_{l=1}^{L} \Delta_{l}=1, \Delta_{l} \geqslant 0
\end{array}\right.
$$

对比式(27)和式(7)可以发现，在求解式(27) 时, 仅需将式(7)中的 $\boldsymbol{x}$ 和 $\boldsymbol{H}$ 替换为 $\boldsymbol{K}(\boldsymbol{x})$ 和 $\tilde{\boldsymbol{\beta}}$. 因 此, 非线性方法可以使用上述算法步骤求解, 在给 定核函数的情况下, 只需将本文方法的输人样本 $\boldsymbol{x}_{i}^{l}$ 和 $\boldsymbol{y}_{j}^{l}$ 替换为 $\boldsymbol{K}\left(\boldsymbol{x}_{i}^{l}\right)$ 和 $\boldsymbol{K}\left(\boldsymbol{y}_{j}^{l}\right)$.

\section{3 实 验}

\section{1 数据集}

实验中使用的是 Google 数据集 ${ }^{[22]}$ 和 WHU-RS 数据集 ${ }^{[23]}$. Google 数据集是一个空中场景数据集, 由 12 类空中场景图像组成, 每类包含 200 幅图像, 每幅图像的大小为 $200 \times 200$ 像素, 每个像素使用 RGB 光谱测量. 图 1 所示为 Google 数据集中的商 业、港口、工业、公园和河流中的示例图像. WHU-RS 数据集也是一个空中场景数据集, 包含 19 类共 950 幅卫星图像, 每个像素使用 RGB 光谱 测量, 每幅图像的大小为 $600 \times 600$ 像素. 图 2 所示 为 WHU-RS 数据集中桥梁、沙漠、足球场、山区 和火车站的示例图像.

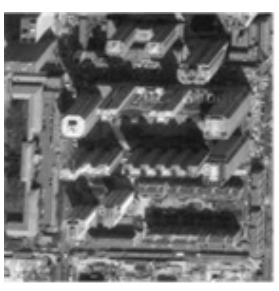

a. 商业

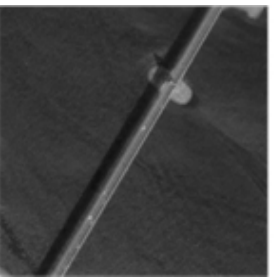

a. 桥梁

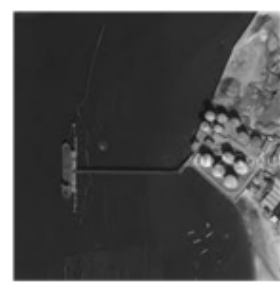

b. 港口

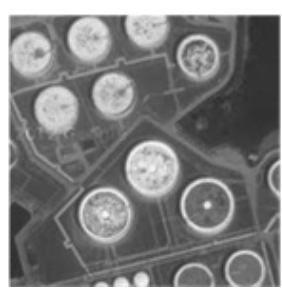

c. 工业

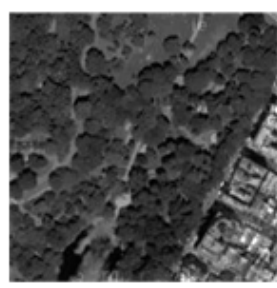

d. 公园

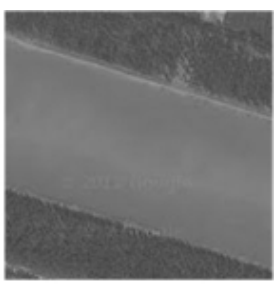

e. 河流

图 1 Google 数据集的示例

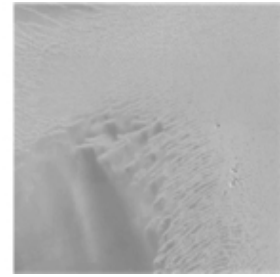

b. 沙漠

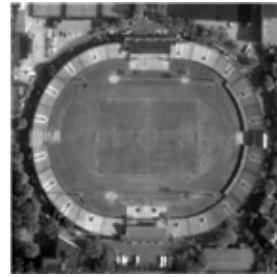

c. 足球场

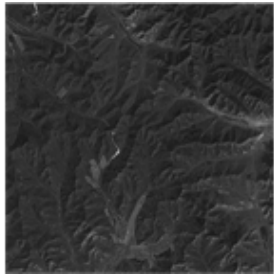

d. 山区

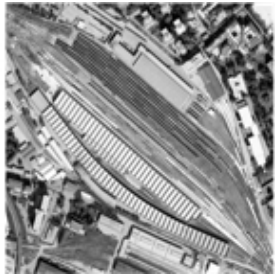

e. 火车站

图 2 WHU-RS 数据集的示例

为了有效地表示遥感图像, 实验采用 2 种不同 的特征表示方法进行遥感场景分类. 一种是传统 的特征表示方法, 包括 LBP, SIFT 和 MR8 (maximum response 8$)^{[24]}$, 得到的特征维数分别是 36 维、 1024 维和 12 维; 另一种是深层数据特征, 由 4 个 不同特征提取层构成的无监督卷积神经网络 (unsupervised convolutional neural networks, $\mathrm{UCNN})^{[25]}$ 提取获得, 4 个 UCNN 分别具有 1 4 个特 征提取层, 特征维数均为 1024 , 得到的深度特征 分别命名为 UCNN1, UCNN2, UCNN3 和 UCNN4. 实验中每个视角使用的不同特征表示，其详细信 息如表 1 所示.
表 1 不同视角使用的特征表示

\begin{tabular}{ccc}
\hline 视角 & 特征表示方法 & 数据维数 \\
\hline 1 & LBP+ SIFT+ MR8 & 1072 \\
2 & UCNN1 & 1024 \\
3 & UCNN2 & 1024 \\
4 & UCNN3 & 1024 \\
5 & UCNN4 & 1024 \\
\hline
\end{tabular}

\section{2 实验及结果分析}

在本文方法的评价实验中，对比了 2 类方法， 即单视角分类方法和多视角分类方法. 3 种单视角 分类方法包括邻域保持嵌人 (neighborhood pre- 
serving embedding, NPE $)^{[26]}$, 最大折叠度量学习 (maximally collapsing metric learning, MCML) ${ }^{[27]}$ 和 信息论度量学习(information-theoretic metric learning, ITML $)^{[28] ;} 3$ 种多视角分类方法包括协作式多 视图度量学习 (collaborative multi-view metric learning, CMML) ${ }^{[29]}$, 多视角核向量机 (multi-view core vector machine, MvCVM) ${ }^{[16]}$ 和多视角 TSK 模 糊系统 (multiview TSK fuzzy system, MV-TSK$F S)^{[17]}$. 对于参数的选取范围, 对比方法均采用原 始文献的默认设置. ITML 的松他变量设置为 $\{0.01$, $0.10,1.00,10.00\}$. CMML 的平衡参数为 1 , 学习率 为 $10^{-6}$, 参数 $p=5$. MV-TSK-FS 中模糊规则设置为 $\{5,10, \cdots, 30\}, 3$ 个正则化参数设置为 $\left\{10^{-2}, 10^{-1}, \cdots\right.$, $\left.10^{2}\right\}$. MvCVM 各视角惩罚因子在网格 $\left\{1,10^{1}, \cdots, 10^{6}\right\}$ 中搜索选取. 设置 SW-MVML 的模糊指数 $r=\{1.1$, $1.5,2,2.5,3.0\}$, 非线性 SW-MVML 的核函数使用 高斯核 $k\left(\boldsymbol{x}_{i}, \boldsymbol{x}_{j}\right)=\exp \left(-\left\|\boldsymbol{x}_{i}-\boldsymbol{x}_{j}\right\|^{2} / \sigma\right)$, 核参数在网 格 $\left\{10^{-2}, 10^{-1}, \cdots, 10^{2}\right\}$ 中搜索选取. 线性和非线性 SW-MVML 都使用 SVM 作为分类器, SVM 的惩罚 因子设置为 $\left\{10^{-3}, 10^{-1}, \cdots, 10^{3}\right\}$. 实验中所有方法 采用网格搜索的方式选择最佳的参数, 运行环境 为 $17-8700 \mathrm{k} 3.2 \mathrm{GHz} \mathrm{CPU}, 32 \mathrm{~GB} \mathrm{RAM}$, 运行软件 为 Matlab 2016. 从 Google 数据集中随机选择 $80 \%$ 的图像进行训练，对剩余 20\%的图像进行测试. 因 为 WHU-RS 数据集每种分类的图像较少，随机选 择 30 个样本进行训练, 并选择 20 个样本进行测试. 评价标准采用分类准确率, 实验执行 10 次并记录 每次结果.

\section{3 线性-非线性 SW-MVML 比较}

首先比较线性-非线性 SW-MVML 在 Google 和 WHU-RS 数据集上的分类性能. 图 3 所示为这 2 种方法在 Google 和 WHU-RS 数据集上的分类准确 率. 可以看到, 非线性 SW-MVML 在 Google 和
WHU-RS 遥感图像数据集上的分类准确率比线性 SW-MVML 分别高 1.2\%和 1.6\%. 图 3 中结果表明, 使用核技术可以提高 SW-MVML 在遥感场景图像 的分类性能.

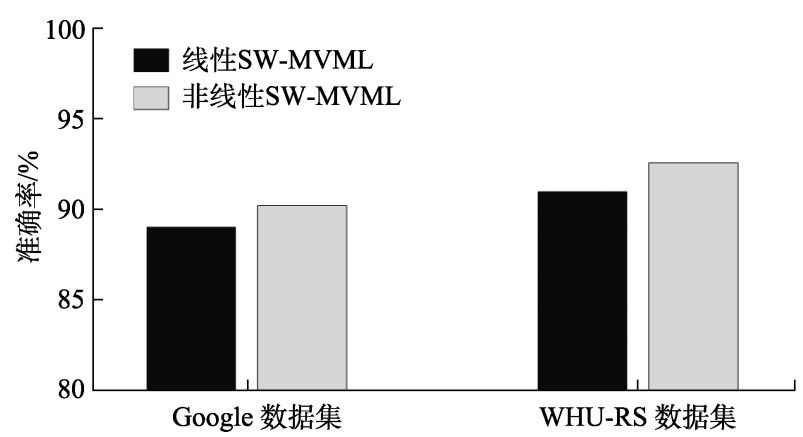

图 3 线性-非线性 SW-MVML 在 2 个数据集上的 分类准确率比较

为了进一步验证非线性 SW-MVML 的分类性 能, 图 4 和图 5 所示为非线性 SW-MVML 在 Google 和 WHU-RS 数据集上的混淆矩阵. 混淆矩阵显示 每个场景类别的分类情况，混淆矩阵中的值表示 对应的类别中分类正确的图像数. Google数据集中 每个场景类别的测试数据包含 40 幅图像. 非线性 SW-MVML 在水、住宅和商业场所中对所有样本均 分类正确, 准确率为 $100 \%$; 在公园、牧场、闲置 土地、工业和农业中的分类准确率超过 $90 \%$. 在 WHU-RS 数据集上，除了桥梁、工业、港口和住宅 的场景类别外，非线性 SW-MVML 对其他 15 个场 景类别的分类准确率超过 $90 \%$. 对于呈现高维非 线性的遥感场景图像，在线性空间中往往不能充 分利用不同视角的数据信息. 通过使用核技术，非 线性 SW-MVML 能更有效地挖掘隐藏于视角间的 关联信息, 进而学习得到潜在于多个视角的共享 空间并获得最优的图像辨识能力. 因此，在后续的 对比实验中，将使用非线性 SW-MVML 与其他方 法进行比较.

\begin{tabular}{|ccccccccccccc|}
\hline & 水 & 河流 & 住宅 & 池塘 & 公园 & 天桥 & 牧场 & 工业 & 闲置土地 & 港口 & 商业 & 农业 \\
水 & 40 & 0 & 0 & 0 & 0 & 0 & 0 & 0 & 0 & 0 & 0 & 0 \\
河流 & 0 & 29 & 0 & 3 & 1 & 0 & 1 & 1 & 1 & 2 & 0 & 0 \\
住宅 & 0 & 0 & 40 & 0 & 0 & 0 & 0 & 0 & 0 & 0 & 0 & 0 \\
池塘 & 0 & 1 & 0 & 31 & 5 & 1 & 0 & 0 & 1 & 0 & 1 & 0 \\
公园 & 0 & 0 & 1 & 0 & 37 & 0 & 2 & 0 & 0 & 0 & 0 & 0 \\
天桥 & 0 & 1 & 0 & 1 & 1 & 33 & 1 & 1 & 2 & 0 & 0 & 0 \\
牧场 & 0 & 0 & 0 & 0 & 1 & 1 & 37 & 0 & 1 & 0 & 0 & 0 \\
工业 & 0 & 0 & 0 & 0 & 0 & 0 & 0 & 38 & 0 & 2 & 0 & 0 \\
闲置土地 & 0 & 0 & 1 & 0 & 1 & 1 & 0 & 0 & 36 & 0 & 0 & 1 \\
港口 & 3 & 0 & 0 & 1 & 0 & 0 & 0 & 0 & 1 & 35 & 0 & 0 \\
商业 & 0 & 0 & 0 & 0 & 0 & 0 & 0 & 0 & 0 & 0 & 40 & 0 \\
农业 & 1 & 0 & 0 & 0 & 0 & 0 & 2 & 0 & 0 & 0 & 0 & 37 \\
\hline
\end{tabular}

图 4 非线性 SW-MVML 在 Google 数据集上的分类结果混淆矩阵 


\begin{tabular}{|c|c|c|c|c|c|c|c|c|c|c|c|c|c|c|c|c|c|c|c|}
\hline & 机场 & 海滩 & 桥 & 商业 & 沙漠 & 农田 & 足球场 & 森林 & 工业 & 牧场 & 山 & 公园 & 停车场 & 池塘 & 港口 & 火车站 & 住宅 & 河流 & 高架桥 \\
\hline 机场 & 18 & 0 & 0 & 0 & 0 & 0 & 0 & 0 & 0 & 0 & 0 & 0 & 0 & 0 & 0 & 0 & 0 & 0 & 2 \\
\hline 海滩 & 0 & 20 & 0 & 0 & 0 & 0 & 0 & 0 & 0 & 0 & 0 & 0 & 0 & 0 & 0 & 0 & 0 & 0 & 0 \\
\hline 桥 & 0 & 0 & 17 & 0 & 0 & 0 & 1 & 0 & 0 & 0 & 0 & 0 & 1 & 0 & 1 & 0 & 0 & 0 & 0 \\
\hline 商业 & 0 & 0 & 0 & 20 & 0 & 0 & 0 & 0 & 0 & 0 & 0 & 0 & 0 & 0 & 0 & 0 & 0 & 0 & 0 \\
\hline 沙漠 & 0 & 0 & 0 & 0 & 20 & 0 & 0 & 0 & 0 & 0 & 0 & 0 & 0 & 0 & 0 & 0 & 0 & 0 & 0 \\
\hline 农田 & 0 & 0 & 0 & 0 & 1 & 18 & 0 & 0 & 0 & 1 & 0 & 0 & 0 & 0 & 0 & 0 & 0 & 0 & 0 \\
\hline 足球场 & 0 & 0 & 0 & 0 & 0 & 0 & 20 & 0 & 0 & 0 & 0 & 0 & 0 & 0 & 0 & 0 & 0 & 0 & 0 \\
\hline 森林 & 0 & 0 & 0 & 0 & 0 & 0 & 0 & 20 & 0 & 0 & 0 & 0 & 0 & 0 & 0 & 0 & 0 & 0 & 0 \\
\hline 工业 & 0 & 0 & 0 & 0 & 0 & 0 & 0 & 0 & 16 & 0 & 0 & 0 & 0 & 0 & 0 & 3 & 1 & 0 & 0 \\
\hline 牧场 & 0 & 0 & 0 & 0 & 0 & 0 & 0 & 1 & 0 & 18 & 1 & 0 & 0 & 0 & 0 & 0 & 0 & 0 & 0 \\
\hline 山 & 0 & 0 & 0 & 0 & 0 & 0 & 0 & 0 & 0 & 0 & 20 & 0 & 0 & 0 & 0 & 0 & 0 & 0 & 0 \\
\hline 公园 & 0 & 0 & 0 & 0 & 0 & 0 & 0 & 0 & 0 & 0 & 0 & 20 & 0 & 0 & 0 & 0 & 0 & 0 & 0 \\
\hline 停车场 & 0 & 0 & 0 & 0 & 0 & 0 & 0 & 0 & 0 & 0 & 0 & 0 & 18 & 0 & 1 & 0 & 1 & 0 & 0 \\
\hline 池塘 & 0 & 0 & 0 & 0 & 0 & 0 & 0 & 0 & 0 & 0 & 0 & 0 & 0 & 19 & 0 & 0 & 0 & 1 & 0 \\
\hline 港口 & 0 & 0 & 1 & 1 & 0 & 0 & 1 & 0 & 1 & 0 & 0 & 0 & 1 & 0 & 15 & 0 & 0 & 0 & 0 \\
\hline 火车站 & 0 & 0 & 0 & 0 & 0 & 0 & 0 & 0 & 0 & 0 & 0 & 0 & 0 & 0 & 0 & 18 & 0 & 0 & 2 \\
\hline 住宅 & 0 & 0 & 0 & 2 & 0 & 0 & 0 & 0 & 1 & 0 & 0 & 0 & 0 & 0 & 0 & 0 & 17 & 0 & 0 \\
\hline 河流 & 0 & 0 & 0 & 0 & 0 & 0 & 0 & 1 & 0 & 0 & 0 & 0 & 0 & 0 & 0 & 0 & 0 & 19 & 0 \\
\hline 高架桥 & 0 & 0 & 0 & 0 & 0 & 0 & 0 & 0 & 0 & 0 & 0 & 0 & 0 & 0 & 1 & 0 & 0 & 0 & 19 \\
\hline
\end{tabular}

图 5 非线性 SW-MVML 在 WHU-RS 数据集上的分类结果混淆矩阵

\section{4 不同方法的性能比较}

本节比较非线性 SW-MVML 与单视角和多视 角分类方法在 Google 和 WHU-RS 数据集的分类准 确率, 实验结果分别如表 2 和表 3 所示. 由表 2 和 表 3 可得出如下结论.

(1) 4 种多视角分类方法的分类准确率均优于 3 种单视角分类方法. 鉴于遥感图像的高维特性, 多视角方法利用不同视角间的互补信息能够更好 地区分不同类别的空间信息，能提高遥感图像场 景的分类准确率.

(2) CMML 方法使用线性投影，而高维遥感场 景图像往往具体非线性的特点，在线性空间的分 类效果不佳. $\mathrm{MvCVM}$ 方法的理论依据是分类间隔 最大化理论，但往往不能充分利用同类别数据间
的信息，对于分布于分类边界的样本也容易分错. 而模糊系统 MV-TSK-FS 虽然能处理多个视角的数 据, 但在处理高维数据时不具有优势.

(3) SW-MVML 使用马氏距离度量, 考虑样本 不同维度的特征之间的关联，其通过计算样本间 的协方差矩阵进行度量, 是一种有效计算 2 个样本 间距离的方式. SW-MVML 中样本间差异越大的图 像对相距越远，样本间差异越小的图像对相距越 近，达到最小化同类图像对间的距离同时最大化 异类图像对间的距离的目的.

（4）非线性 SW-MVML 在所有场景类别中都 实现最佳的分类性能. 因为非线性 SW-MVML 通 过多视角度量学习能同时利用多个视角的数据特 征，同时在多视角协同学习的基础上考虑视角加

表 2 Google 数据集上不同方法的分类准确率比较

\begin{tabular}{|c|c|c|c|c|c|c|c|}
\hline 类别 & $\mathrm{NPE}^{[26]}$ & $\mathrm{MCML}^{[27]}$ & $\mathrm{ITML}^{[28]}$ & $\mathrm{CMML}^{[29]}$ & $\operatorname{MvCVM}^{[16]}$ & MV-TSK-FS ${ }^{[17]}$ & 非线性 SW-MVML \\
\hline 水 & 96.63 & 96.65 & 96.75 & 97.34 & 97.26 & 97.80 & 99.21 \\
\hline 河流 & 69.69 & 69.75 & 69.88 & 70.84 & 70.25 & 70.93 & 72.64 \\
\hline 住宅 & 95.89 & 95.92 & 95.89 & 96.93 & 96.88 & 97.01 & 99.66 \\
\hline 池塘 & 73.25 & 73.52 & 73.58 & 76.07 & 76.34 & 76.67 & 77.79 \\
\hline 公园 & 88.22 & 88.32 & 88.36 & 89.40 & 89.59 & 90.44 & 92.67 \\
\hline 天桥 & 78.61 & 78.77 & 78.72 & 79.69 & 79.59 & 80.73 & 82.52 \\
\hline 草地 & 88.12 & 88.29 & 88.41 & 89.45 & 89.63 & 90.46 & 92.63 \\
\hline 工业 & 90.73 & 90.65 & 90.85 & 94.92 & 94.96 & 94.94 & 95.75 \\
\hline 闲置土地 & 85.96 & 85.05 & 85.14 & 86.19 & 86.04 & 87.21 & 89.97 \\
\hline 港口 & 84.26 & 84.29 & 84.35 & 86.51 & 86.68 & 87.52 & 87.82 \\
\hline 商业 & 95.38 & 95.35 & 95.43 & 97.57 & 97.63 & 97.64 & 99.85 \\
\hline 农业 & 88.87 & 88.85 & 88.96 & 91.02 & 91.24 & 92.03 & 92.63 \\
\hline 平均值 & 86.30 & 86.28 & 86.36 & 87.99 & 88.01 & 88.62 & 90.26 \\
\hline
\end{tabular}

注. 粗体表示准确率最高. 
表 3 WHU-RS 数据集上不同方法的分类准确率比较

$\%$

\begin{tabular}{|c|c|c|c|c|c|c|c|}
\hline 类别 & $N P E^{[26]}$ & $\mathrm{MCML}^{[27]}$ & ITML $^{[28]}$ & $\mathrm{CMML}^{[29]}$ & $\mathrm{MvCVM}^{[16]}$ & MV-TSK-FS ${ }^{[17]}$ & 非线性 SW-MVML \\
\hline 机场 & 87.68 & 87.59 & 87.72 & 88.75 & 89.05 & 89.79 & 91.86 \\
\hline 海滩 & 95.25 & 95.56 & 95.71 & 96.83 & 97.10 & 96.86 & 98.92 \\
\hline 桥 & 82.21 & 82.33 & 82.55 & 83.69 & 83.90 & 84.78 & 85.77 \\
\hline 商业 & 94.99 & 95.21 & 95.19 & 97.08 & 97.55 & 97.14 & 99.20 \\
\hline 沙漠 & 94.86 & 94.93 & 94.85 & 97.87 & 97.15 & 97.88 & 98.96 \\
\hline 农田 & 86.36 & 86.56 & 86.69 & 87.75 & 87.08 & 87.78 & 89.65 \\
\hline 足球场 & 94.52 & 94.68 & 94.75 & 96.71 & 96.14 & 96.78 & 98.00 \\
\hline 森林 & 94.69 & 94.85 & 94.79 & 97.82 & 98.13 & 97.82 & 99.89 \\
\hline 工业 & 77.28 & 77.36 & 77.58 & 78.69 & 78.96 & 78.70 & 80.59 \\
\hline 草地 & 88.17 & 86.83 & 87.22 & 88.31 & 88.61 & 89.40 & 91.36 \\
\hline 山 & 94.82 & 94.88 & 94.93 & 96.87 & 96.24 & 96.88 & 98.92 \\
\hline 公园 & 95.30 & 95.56 & 95.77 & 97.61 & 97.10 & 97.68 & 98.85 \\
\hline 停车场 & 85.22 & 85.43 & 85.44 & 88.65 & 88.83 & 88.72 & 89.98 \\
\hline 池塘 & 90.99 & 91.05 & 91.11 & 92.24 & 92.44 & 92.24 & 94.87 \\
\hline 港口 & 71.87 & 71.95 & 71.88 & 73.82 & 74.23 & 73.87 & 75.22 \\
\hline 铁路站 & 85.20 & 85.37 & 85.51 & 88.62 & 88.90 & 88.70 & 90.92 \\
\hline 住宅 & 81.59 & 81.58 & 81.36 & 82.88 & 83.73 & 83.98 & 85.09 \\
\hline 河流 & 92.81 & 92.79 & 92.93 & 93.85 & 93.25 & 93.87 & 95.96 \\
\hline 高架桥 & 91.63 & 92.13 & 92.28 & 93.15 & 94.66 & 94.15 & 95.77 \\
\hline 平均值 & 88.71 & 88.77 & 88.86 & 90.59 & 90.69 & 90.90 & 92.62 \\
\hline
\end{tabular}

注. 粗体表示准确率最高.

权，模型在迭代优化中能够自适应地调整各视角 的权重，并同时达到最优的图像分辨能力. 另外， 通过使用核技术，非线性 SW-MVML 能更有效地 挖掘隐藏于视角间的关联信息，使其获取的图像 分辨能力更准确.

综合以上实验结果，可以得到 SW-MVML 适 用于遥感场景分类的结论.

\section{5 参数分析}

非线性 SW-MVML 涉及的参数为模糊指数 $r$ 和核参数 $\sigma$, 以及 SVM 分类器的惩罚因子 $C$; 所 有参数均在实验设定的范围内寻优获得. 表 4 表 6 分别显示参数 $r, \sigma$ 和 $C$ 在 Google 和 WHU-RS 数 据集上对分类准确率的影响. 从表 4 表 6 可以得到 以下结论.

(1) 模糊指数 $r$ 到调节视角权重的作用, $r$ 的值 越大，视角间的权重越接近; $r$ 的值越小，视角间 的权重差异就越大. 从表 4 的结果可以看出, 模糊 指数 $r$ 对分类准确率的影响是温和的. 因此，在实 验中可以选定 $r=2$.

(2) 通过核函数，原始数据样本被映射到特征 空间，核参数 $\sigma$ 决定非线性 SW-MVML 运行的特 征空间. 从表 5 的结果可以看出，核参数 $\sigma$ 对分类 准确率起到决定性作用. 不同 $\sigma$ 下 SW-MVML 取
得不同的分类准确率, 且差异较大. 因此, 在实验 中对 $\sigma$ 使用网络搜索法寻优是合理的.

(3) 惩罚因子 $C$ 起到平衡 SVM 分类器中经验 误差项和结构误差项的作用. 特征空间下 $C$ 的取 值小表示经验误差项的比例小而结构误差项的比 例大; $C$ 的取值大表示经验误差项的比例大而结构 误差项的比例小. 从表 6 的结果可以看出, $C$ 的取 值也对分类准确率起到决定性作用，不同 $C$ 值得

表 4 非线性 SW-MVML 的参数 $r$ 在 2 个数据集上 分类准确率比较

\begin{tabular}{lccccc}
\hline \multirow{2}{*}{ 数据集 } & \multicolumn{5}{c}{$r$} \\
\cline { 2 - 6 } & 1.1 & 1.5 & 2.0 & 2.5 & 3.0 \\
\hline Google & 90.01 & 90.14 & $\mathbf{9 0 . 2 6}$ & 90.04 & 90.01 \\
WHU-RS & 92.25 & 92.40 & $\mathbf{9 2 . 6 2}$ & 92.50 & 92.38 \\
\hline
\end{tabular}

注. 粗体表示准确率最高.

表 5 非线性 SW-MVML 的参数 $\sigma$ 在 2 个数据集上 分类准确率比较

\begin{tabular}{lccccc}
\hline \multirow{2}{*}{ 数据集 } & \multicolumn{5}{c}{$\sigma$} \\
\cline { 2 - 6 } & $10^{-2}$ & $10^{-1}$ & 1 & 10 & $10^{2}$ \\
\hline Google & 88.03 & $\mathbf{9 0 . 2 6}$ & 89.65 & 84.08 & 80.74 \\
WHU-RS & 84.28 & 90.59 & $\mathbf{9 2 . 6 2}$ & 87.70 & 80.81 \\
\hline
\end{tabular}

注. 粗体表示准确率最高. 
表 6 非线性 SW-MVML 的参数 $C$ 在 2 个数据集上 分类准确率比较

\section{$\%$}

\begin{tabular}{lccccccc}
\hline \multirow{2}{*}{ 数据集 } & \multicolumn{8}{c}{$C$} \\
\cline { 2 - 8 } & $10^{-3}$ & $10^{-2}$ & $10^{-1}$ & 1 & 10 & $10^{2}$ & $10^{3}$ \\
\hline Google & 73.67 & 75.34 & 80.57 & 89.22 & $\mathbf{9 0 . 2 6}$ & 87.53 & 85.14 \\
WHU-RS & 80.05 & 84.67 & 87.02 & $\mathbf{9 2 . 6 2}$ & 89.81 & 86.07 & 85.89 \\
\hline
\end{tabular}

注. 粗体表示准确率最高.

到的分类准确率差异较大. 因此, 在实验中 $C$ 使用 网络搜索法寻优也是必要的.

另外, 图 6 所示为 SW-MVML 方法的 5 个视 角在 Google 和 WHU-RS 数据集上的权重. 在这 2 个数据集上，视角 4 和视角 5 的权重均较大，视角 1 的权重最小, 说明非线性 SW-MVML 分类准确率 最优时深度特征较传统特征权重更大. 同时, 5 个视 角数据均赋予不同的权重，说明非线性 SW-MVML 方法能自适应地找出视角的最佳权重，在 5 个视角 数据的共同作用下，具有最优分辨能力.
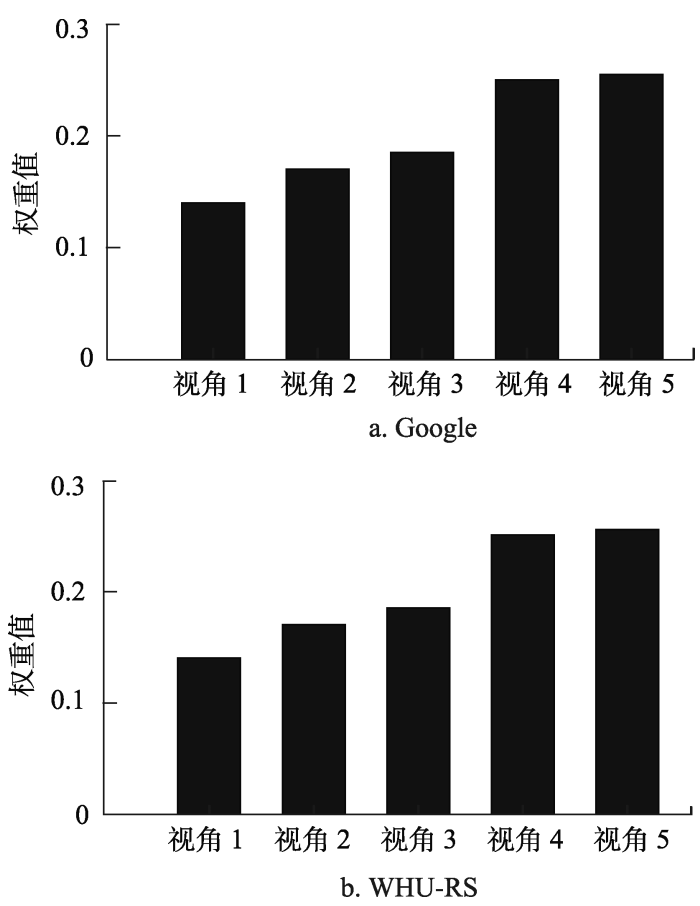

图 6 非线性 SW-MVML 在 2 个数据集上各视角权重情况

\section{4 结 语}

本文提出一种多视角度量学习方法用于遥感 图像的场景分类. 通过充分利用多个视角间的关 联性和差异性，学习一种判别度量空间，以使同类 图像紧凑和异类图像尽可能分离. 将遥感图像投 影到 RKHS 中的判别空间，本文方法显示出更佳 的分类性能.下一阶段，拟从以下几个方面对本文
方法进行提升：首先，使用梯度下降法求解模型， 如何提高算法的执行效率是下阶段研究的方向; 其次，如何更有效地选择核参数也是值得研究的 问题; 同时，考虑将类内紧凑性和类间分离性作为 分类的判别信息. 接下来考虑将其他区分性概念 使用到本文的多视角度量学习的框架中.

\section{参考文献(References):}

[1] Li C, Gao L R, Plaza A, et al. FPGA implementation of a maximum simplex volume algorithm for endmember extraction from remotely sensed hyperspectral images[J]. Journal of Real-Time Image Processing, 2019, 16(5): 1681-1694

[2] Xie J, He N J, Fang L Y, et al. Scale-free convolutional neural network for remote sensing scene classification[J]. IEEE Transactions on Geoscience and Remote Sensing, 2019, 57(9): 6916-6928

[3] Yang Haoqi, Yao Hongge, Wang Cheng, et al. Detection and segmentation and positioning of remote sensing ships in complex scenes[J]. Journal of Computer-Aided Design \& Computer Graphics, 2020, 32(3): 472-485(in Chinese)

(杨浩琪, 姚红革, 王诚, 等. 复杂场景下遥感船舶的检测与 分割定位 $[\mathrm{J}]$. 计算机辅助设计与图形学学报, 2020, 32(3): 472-485)

[4] Xu K J, Huang H, Deng P F, et al. Two-stream feature aggregation deep neural network for scene classification of remote sensing images[J]. Information Sciences, 2020, 539(10): 250-268

[5] Cheng G, Xie X X, Han J W, et al. Remote sensing image scene classification meets deep learning: challenges, methods, benchmarks, and opportunities[J]. IEEE Journal of Selected Topics in Applied Earth Observations and Remote Sensing, 2020, 13(6): 3735-3756

[6] Ojala T, Pietikainen M, Maenpaa T. Multiresolution gray-scale and rotation invariant texture classification with local binary patterns[J]. IEEE Transactions on Pattern Analysis and Machine Intelligence, 2002, 24(7): 971-987

[7] Zhong Weifeng, Guo Feng, Xiang Shiming, et al. Ship detection in remote sensing based with rotated rectangular region[J]. Journal of Computer-Aided Design \& Computer Graphics, 2019, 31(11): 1935-1945(in Chinese)

(仲伟峰, 郭峰, 向世明, 等. 旋转矩形区域的遥感图像舰船 目标检测模型 $[J]$. 计算机辅助设计与图形学学报, 2019, 31(11): 1935-1945)

[8] Chen C, Zhang B C, Su H J, et al. Land-use scene classification using multi-scale completed local binary patterns[J]. Signal, Image and Video Processing, 2016, 10(4): 745-752

[9] Xu X, Li J, Li S T. Multiview intensity-based active learning for hyperspectral image classification[J]. IEEE Transactions on Geoscience and Remote Sensing, 2018, 56(2): 669-680

[10] Zhou X, Prasad S, Crawford M M. Wavelet-domain multiview active learning for spatial-spectral hyperspectral image classification[J]. IEEE Journal of Selected Topics in Applied Earth Observations and Remote Sensing, 2016, 9(9): 4047-4059 
[11] Luus F P S, Salmon B P, van den Bergh F, et al. Multiview deep learning for land-use classification[J]. IEEE Geoscience and Remote Sensing Letters, 2015, 12(12): 2448-2452

[12] Yang X H, Liu W F, Tao D P, et al. Canonical correlation analysis networks for two-view image recognition[J]. Information Sciences, 2017, 385(4): 338-352

[13] Lefèvre S, Tuia D, Wegner J D, et al. Toward seamless multiview scene analysis from satellite to street level[J]. Proceedings of the IEEE, 2017, 105(10): 1884-1899

[14] Liu Bin, Chen Xiangning, Guo Lianpeng. Research on fast automatic stitching algorithm of massive UAV remote sensing images[J]. Engineering Journal of Wuhan University, 2015, 48(2): 263-268(in Chinese)

(刘涁, 陈向宁, 郭连朋. 无人机海量遥感影像快速自动拼 接算法研究 [J]. 武汉大学学报: 工学版, 2015, 48(2): 263-268)

[15] Wu Fangqing, Yang Yang, Pan Anning, et al. Multi-viewpoints remote sensing images registration using mixed features[J]. Journal of Image and Graphics, 2017, 22(8): 1154-1161(in Chinese)

(吴芳青，杨扬，潘安宁，等. 利用混合特征的多视角遥感图 像配准[J]. 中国图象图形学报, 2017, 22(8):1154-1161)

[16] Huang C Q, Chung F L, Wang S T. Multi-view L2-SVM and its multi-view core vector machine[J]. Neural Networks, 2016, 75: 110-125

[17] Jiang Y Z, Deng Z H, Chung F L, et al. Recognition of epileptic EEG signals using a novel multiview TSK fuzzy system[J]. IEEE Transactions on Fuzzy Systems, 2017, 25(1): 3-20

[18] Bertini E, Tatu A, Keim D. Quality metrics in high-dimensional data visualization: an overview and systematization[J]. IEEE Transactions on Visualization and Computer Graphics, 2011, 17(12): 2203-2212

[19] Ma L, Liu Y, Zhang X L, et al. Deep learning in remote sensing applications: A meta-analysis and review[J]. ISPRS Journal of Photogrammetry and Remote Sensing, 2019, 152(6): 166-177

[20] Bachmann C M, Ainsworth T L, Fusina R A. Exploiting mani- fold geometry in hyperspectral imagery[J]. IEEE Transactions on Geoscience and Remote Sensing, 2005, 43(3): 441-454

[21] Chen G B, Zhang Y, Wang S L. Hyperspectral remote sensing IQA via learning multiple kernels from mid-level features[J]. Signal Processing: Image Communication, 2020, 83(4): 115804

[22] Zhu Q Q, Zhong Y F, Zhao B, et al. Bag-of-visual-words scene classifier with local and global features for high spatial resolution remote sensing imagery[J]. IEEE Geoscience and Remote Sensing Letters, 2016, 13(6): 747-751

[23] Xia G S, Yang W, Delon J, et al. Structural high-resolution satellite image indexing[C] //Proceedings of the ISPRS, TC VII Symposium Part A: 100 Years ISPRS-Advancing Remote Sensing Science. Vienna: ISPRS Press, 2010: 298-303

[24] Varma M, Zisserman A. A statistical approach to texture classification from single images[J]. International Journal of Computer Vision, 2005, 62(1): 61-81

[25] Li Y S, Zhang Y J, Tao C, et al. Content-based high-resolution remote sensing image retrieval via unsupervised feature learning and collaborative affinity metric fusion[J]. Remote Sensing, 2016, 8(9): Article No.709

[26] Wen J H, Tian Z, She H W, et al. Feature extraction of hyperspectral images based on preserving neighborhood discriminant embedding[C] //Proceedings of the International Conference on Image Analysis and Signal Processing. Los Alamitos: IEEE Computer Society Press, 2010: 11357905

[27] Globerson A, Roweis S. Metric learning by collapsing classes[C] //Proceedings of Advances in Neural Information Processing Systems. Cambridge: MIT Press, 2005: 451-458

[28] Davis J V, Kulis B, Jain P, et al. Information-theoretic metric learning[C] //Proceedings of the 24th International Conference on Machine Learning. New York: ACM Press, 2007: 209-216

[29] Hu J L, Lu J W, Yuan J S, et al. Collaborative multi-view metric learning for visual classification[C] //Proceedings of the IEEE International Conference on Multimedia and Expo. Los Alamitos: IEEE Computer Society Press, 2016: 1-6 